\title{
Quantum chaos in one dimension?
}

\author{
László Ujfalusi and Imre Varga \\ Elméleti Fizika Tanszék, Fizikai Intézet, Budapesti Müszaki és Gazdaságtudományi Egyetem, H-1521 Budapest, Hungary \\ Dániel Schumayer \\ Department of Physics, University of Otago, 730 Cumberland Street, Dunedin 9016, New Zealand
}

(Dated: November 12, 2018)

\begin{abstract}
In this work we investigate the inverse of the celebrated Bohigas-Giannoni-Schmit conjecture. Using two inversion methods we compute a one-dimensional potential whose lowest $N$ eigenvalues obey random matrix statistics. Our numerical results indicate that in the asymptotic limit, $N \rightarrow$ $\infty$, the solution is nowhere differentiable and most probably nowhere continuous. Thus such a counterexample does not exist.
\end{abstract}

PACS numbers: 05.45.Mt, 02.30.Zz, 03.65.Ge

\section{INTRODUCTION}

One of the main milestones of quantum chaos is the celebrated Bohigas-Giannoni-Schmit (BGS) conjecture [1]. This conjecture forms a link between the statistical properties of the spectra of quantum systems with chaotic classical limit and random matrix theory (RMT): "Spectra of time-reversal-invariant systems whose classical analogs are $K$-systems show the same fluctuation properties as predicted by GOE [g]."

This conjecture establishes a connection between chaotic systems and RMT, i.e. Chaos $\rightarrow$ RMT. A number of numerical evidences corroborate this conjecture 3], but a complete analytical proof is still missing. As pointed out in Ref. [4] this universality is a consequence of the properties of the long periodic orbits whose semiclassical limit is also universal. The intimate relation between RMT statistics and its breakdown over energy scales of $\mathcal{O}(h / T)$ where $h$ is Planck's constant and $T$ is the period of the shortest periodic orbit, was emphasized in Refs. [4, 5]. The first attempt to prove the BGS conjectures is due to Andreev et al. [6, 7] but later it turned out to be incomplete. More recently the link between chaotic systems and RMT has been investigated using an improved semiclassical technique $8-10]$. Finally we have to mention that certain chaotic models show Poissonstatistics [11] allowing for the possibility of exceptions, as well.

Hence it is indeed very difficult to understand the exact relationship between quantum chaos and RMT. Certainly it should be helpful to investigate the inverse BGS conjecture, i.e. does the presence of GOE level statistics ever lead to chaos in the classical limit? This would be the opposite link: RMT $\stackrel{?}{\rightarrow}$ Chaos. This question has been raised first by $\mathrm{Wu}$ et al. [12]. The authors' aim was to find a counterexample, i.e. a one-dimensional quantum system with GOE level statistics. However, a one-dimensional system is always integrable guaran-

\footnotetext{
* Contact: varga@phy.bme.hu
}

teed by Liouville's theorem [13]. The choice of $d=1$ is merely because it is easier to do computation in one spatial dimension as compared to $d=2$ or $d=3$. In addition the average level separation is of the order of $\mathcal{O}\left(h^{d}\right)$ which allows a for semiclassical regime in $d>1$ only. Despite the simplicity of $d=1$ the task is not at all easy, finding a potential with a given spectrum leads to an inverse Schrödinger problem. There is almost no chance to achieve this analytically, therefore $\mathrm{Wu}$ et al. used a numerical method to find a one-dimensional potential with the first $N$ energy levels being predefined. Their process gave accurate results for the case of GOE level statistics, hence they concluded that there is an integrable system with GOE level statistics, and provided a counterexample to the inverse BGS conjecture. Wu and Sprung later carried out a similar calculation 14 for a particular, asymptotically GUE spectrum represented by the non-trivial zeros of the Riemann zeta function (see also [15 19]). Their findings led to the concept of a fractal potential, which may produce a chaotic spectrum.

Contrary to $\mathrm{Wu}$ et al. we can only state that the first $N$ eigenvalues of the potential obtained numerically show random matrix type fluctuations leaving any other type of behavior for the remaining infinite number of eigenvalues. Therefore we re-investigate this problem more carefully with the emphasis on the $N \rightarrow \infty$, limit.

We examine certain statistical properties of the resulting potential and conclude that no classical system can be associated with the constructed quantum potential. Our analysis not only confirms a kind of fractal nature of the potential, already conjectured by $\mathrm{Wu}$ and Sprung [12, 16], but exceeds it by showing that the potential asymptotically loses its continuity and differentiability and resembles the sum of randomly positioned Dirac-delta functions.

The structure of our article reads as follows. In Section II we describe the numerical methods applied, Section III compares the results provided by the numerical methods. In Section IV we analyse the $N \rightarrow \infty$ limit and Section $\mathrm{V}$ is left for conclusions. 


\section{THE NUMERICAL METHODS AND THEIR PROPERTIES}

We discuss the inverse BGS conjecture and start by calculating all eigenvalues of a sufficiently large, real, symmetric matrix, thereby creating a GOE spectrum. This raw spectrum has to be unfolded in order to guarantee uniformity [20]. As a result of this procedure, the average distance between two neighboring levels in the unfolded spectrum is unity. The first $N$ members of this unfolded spectrum are thereafter denoted by $e_{1}, \cdots, e_{N}$ and remain unchanged.

We apply two techniques, the numerical method used also by $\mathrm{Wu}$ et al., and the dressing-transformation 15, 18, 21] in order to find a potential, whose first $N$ levels are as close as possible to $e_{1}, \cdots, e_{N}$. By changing $N$ we tried to conclude about the $N \rightarrow \infty$ limit, since in that case the entire spectrum should show GOE properties. Naturally, infinitely many potentials may have the same first $N$ levels, nevertheless their shapes coincide for the energy range determined by the prescribed spectrum and differ only at higher energies.

Below we describe the numerical techniques; an optimisation and an iterative one. These two approaches provide qualitatively and quantitatively similar results concerning the most important properties of the potential.

At first we implemented the same variational algorithm introduced by $\mathrm{Wu}$ et al. 12. This method starts with an initial guess $V_{0}(x)$ for the yet unknown potential $V(x)$ and one calculates the energy eigenvalues of $V_{0}(x)$, which are denoted by $\varepsilon_{1}, \cdots, \varepsilon_{N}$. This spectrum may differ from the pre-defined energy levels, therefore in the next step the potential is altered in a way, that its energy eigenvalues fit the expected spectrum, $e_{1}, \cdots, e_{N}$, better. The same procedure can be continued until convergence is reached.

This method requires an objective function, $G$, measuring the distance between the actual and the predefined spectrum. One may choose $G$ to be

$$
G[V(x)]=\sum_{i=1}^{N}\left(\varepsilon_{i}-e_{i}\right)^{2} .
$$

$G$ implicitly depends on $V(x)$ through the set of eigenvalues, $\varepsilon_{i}$, therefore the task is to minimize $G$ through the variation of $V(x)$. Moreover, $G$ is positive semidefinite, and equals zero if and only if $\varepsilon_{i}=e_{i}(i=1,2, \ldots, N)$.

Employing a standard gradient method the potential has to be updated as

$$
V_{\text {new }}(x)=V_{\text {old }}(x)-2 \eta \sum_{i=1}^{N}\left(\varepsilon_{i}-e_{i}\right)\left[\Phi_{i}(x)\right]^{2} .
$$

If the initial potential, $V_{0}(x)$, is well chosen, the algorithm converges and provides us with a potential, whose first $N$ eigenvalues are very close to the pre-defined ones, $\varepsilon_{i} \approx e_{i}$.
The average distance in the unfolded spectrum of a large GOE matrix is unity, which resembles a harmonic oscillator, therefore a parabolic form seems to be a good choice for $V_{0}(x)$.

Our other method is based on an interesting property of the Schrödinger equation: a bare free quantum system can be "dressed up" in an iterative manner, with a series of potentials incorporating more and more bound states into the system. Thereby this method is generally called dressing transformation 15, 18, 21]. Eventually this algorithm provides a potential, whose first $N$ lowest energy eigenstates are bound states with the pre-defined energy levels, while all other states are scattering states, i.e. $e_{1}<e_{2}<\cdots<e_{N}<0$, and $e_{k}=0$ for $k>N$. One may interpret this method differently, and say that it shifts $N$ scattering states to negative energies, converting them into bound states, while preserving the original difference between the levels. It is important to mention here, that theoretically this method is exact, and only the implementation may introduce numerical error into the final solution.

In the remaining part of this section we comment on the uniqueness of the potential, on the speed of the algorithms, and finally on the numerical error accepted in our calculations.

Uniqueness: If one intends to explore the interaction in a quantum problem by analyzing scattering data, it is a crucial question whether the experimental scattering data is compatible with many different potentials, or just with one. For a generic non-relativistic, onedimensional quantum system Borg has rigorously proven that one spectrum (eigenvalues for the bound states and reflection coefficient for the scattering states) is not sufficient to uniquely reconstruct the corresponding potential [22]. However, from two spectra (obtained using two different boundary conditions) one can determine the potential uniquely. Restricting ourselves to even potentials only, $V(x)=V(-x)$, one spectrum becomes sufficient to uniquely determine the potential [23]. Unfortunately, noone can use an infinite set of spectrum in a numerical algorithm, therefore uniqueness is lost apparently. Our physical intuition suggests though, that if two potentials support the same bound states, then these potentials ought to be identical at least around their bottoms.

This expectation is valid and justified numerically in our calculation. The potential generated by the $\mathrm{Wu}-$ method and by the dressing transform - after an insignificant energy shift - differ only at their "semiclassical edges" which can be characterized by $x_{\text {turn }}$, where $V\left(x_{\text {turn }}\right)=e_{N}$, i.e. where a classical particle with energy $e_{N}$ would turn back in the given potential.

The difference between the potentials is expected outside the $\left[-x_{\text {turn }}, x_{\text {turn }}\right]$ interval, since the Wu-method should reproduce the parabolic potential characteristic of the harmonic oscillator, while in this form of the dressing transformation the first derivative of the potential must approach zero asymptotically, therefore the potential becomes constant. 
Speed: According to our experience the Wu method was very accurate; the error decreases rapidly in each consecutive iteration. Despite its precision, it becomes slow for increasing $N$ mainly due to the repeated calculation of every eigenstates, $\Phi_{i}(x)(i=1,2, \ldots, N)$. This method seems to be prohibitively impractical for $N$ being higher than few hundreds. On the contrary the dressing transformation is very fast, but for large $N$, its accuracy decreased considerably.

Numerical error: Both methods introduce a numerical error due to the discretization. It is, therefore, necessary to check whether the potential calculated reproduces the spectrum prescribed. If the spectrum $\varepsilon_{i}$ does not match the original set of eigenvalues, $e_{i}$, the appropriate parameters of the algorithms must be adjusted: the variational step size, $\eta$, in case of the Wu-method, and the discretization step-size of the Runge-Kutta method for the dressing transformation.

We stopped the algorithms when the average error on each energy level has been reduced below a fixed threshold, namely

$$
\frac{1}{N} \sum_{i=1}^{N}\left|e_{i}-\varepsilon_{i}\right|<10^{-5} .
$$

This seemingly acceptable order of numerical error is easily met by the Wu-method, but represents a stringent condition for the dressing transform, the later of which needed small step-size, $\sim 2 \times 10^{-4}$, for relatively high values of $N$. We have also investigated the accumulation of error and have found that the sum in Eq. (3) collects the main contributions at high energies, i.e. where the two methods are expected to deviate from each other.

\section{RESULTS}

This section provides the results. First let us take a look at Fig. 1 which depicts the potentials obtained by the Wu-method and the dressing transformation for $N=$ 50 .

Note that the two methods do indeed give very similar, symmetric potentials, as we expected based on Borg's theorem. The dressing transformation provides a symmetric potential a priori, while the Wu method is started from a symmetric potential which property is inherited in each iteration. More importantly, one may separate the potential into two parts; a parabola and an oscillatory component, the latter of which will be called fluctuation.

$$
V(x)=\overline{V(x)}+V_{\mathrm{fl}}(x) .
$$

Due to the GOE level fluctuations of the first $N$ eigenvalues the parabola becomes oscillatory in a finite region that is connected to the $N$ th level, i.e. to the turning point $x_{\text {turn }}$ for which $V\left(x_{\text {turn }}\right)=E$. In quantum mechanics the wave function approaches zero very rapidly for $|x|>x_{\text {turn }}$, thus the oscillatory region is simply the

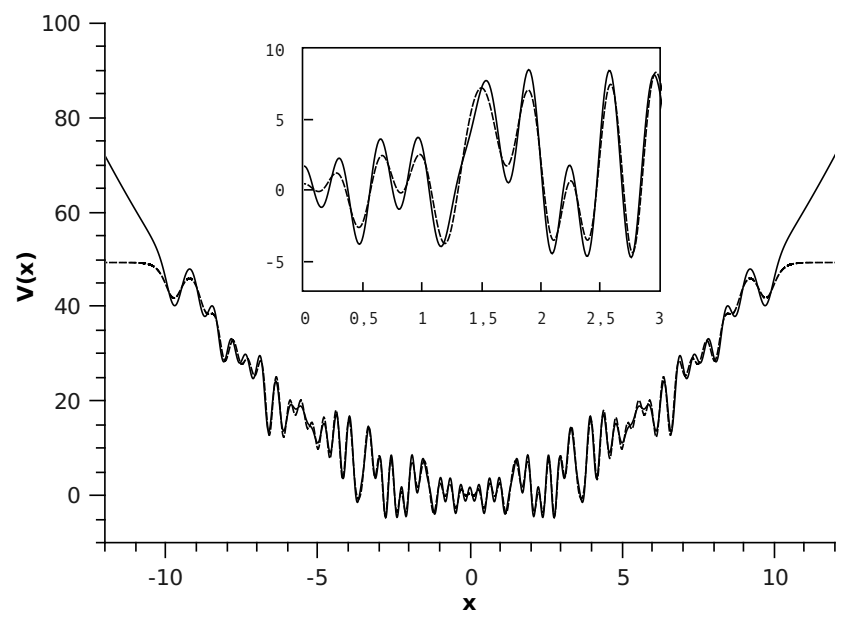

FIG. 1. The potentials calculated with the Wu-method (solid line) and the dressing transformation (dashed line) for $N=50$ are shown. The potentials provided by these methods are almost identical in the $[-10,10]$ range. Inset shows the same potentials in the $[0,3]$ interval.

interval of $\left[-x_{\text {turn }} ; x_{\text {turn }}\right]$ if $E=\varepsilon_{N}$. Hence the average potential becomes wavy in the region according to $x_{\text {turn }}$ corresponding to the $N$-th level. By increasing $N$, the turning point, $x_{\text {turn }}$, increases too, therefore in the $N \rightarrow \infty$ limit the whole potential is expected to be wildly oscillatory. This expectation is supported by Fig. 2 although the largest value of $N$ used is only 250 . However, not only the width of the oscillatory region increases, but the amplitude of the fluctuation term seems to be larger and larger, see Figs. 2 and 3(a). In the following we wish

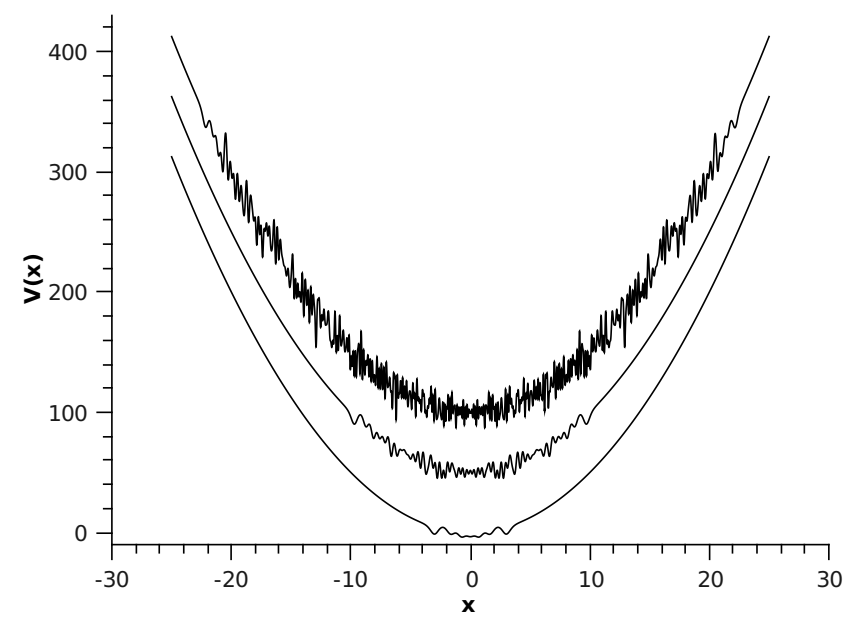

FIG. 2. The potential calculated with the Wu method for $N=5$ (bottom), 50 (middle) and 250 (top). The graphs for $N=50$ and $N=250$ are shifted vertically.

to classify the oscillatory component, $V_{\mathrm{fl}}(x)$, by measur- 
ing the density of the fluctuations. For this reason the distance between two consecutive extrema could serve as a good measure, see Fig. 3(b), therefore, we defined $\Delta x$ and $\Delta y$ as the distances between two adjacent extrema in the $x$ and $y$ directions. For a fixed value of $N$, the average value of $\Delta x$ and $\Delta y$ characterizes the fluctuation of $V(x)$. The average of $\Delta x$ is plotted in Figure 4 for both methods. The graphs suggest a power-law dependence on $N$. The least-squares fit, using $\overline{\Delta x}=A+B N^{-C}$ is also plotted to guide the eye. Provided this dependence is suitable also in the asymptotic limit, $N \rightarrow \infty$, the average width of oscillation in $V_{\mathrm{fl}}(x)$ approaches zero, since $A \approx 0$, while the average amplitude of fluctuation, $\overline{\Delta y}$, remains positive, see Fig. 5] This asymptotic behavior naturally poses the question: does the potential remain differentiable as $N \rightarrow \infty$ ? In order to answer this question we introduce a new quantity with the following definition: $c=\Delta y / \Delta x$. This fraction, at any finite $N$, is not equal to the usual difference fraction, because neither $\Delta x$, nor $\Delta y$ is allowed to change continuously as in calculus, but here they have a finite and fixed local value. For any potential calculated for a fixed $N$, we have a finite set of $c$ values to analyze statistically. In Figure 6] we show the histogram of $c$ for different values of $N$. One can see that this histogram broadens as $N$ increases. Conclusively, $\bar{c}$, the average of $c$, diverges proportional to $N$, which is seen in Fig. 7 The behavior of $\overline{\Delta x}$ and $\bar{c}$ described above allows us to conclude: in the $N \rightarrow \infty$ limit the whole potential will be rapidly oscillatory, because $x_{\text {turn }}$ is growing with $N$. Considering any finite interval of $x, \overline{\Delta x} \rightarrow 0$ as $N \rightarrow \infty$, therefore $c$ becomes the derivative of $V(x)$. From the numerical observations mentioned above one can conclude $(i)$ there are infinitely many extrema, thus infinitely many values of $c$ in a finite interval of $x$, and $(i i)$ a finite part of the $c$-s, still infinitely many, diverge with increasing $N$, since $\bar{c}$ diverges as well. These findings suggest that the potential is nowhere differentiable. Such a potential cannot be compatible with the Hamiltonian formalism of classical mechanics, especially with the Hamilton-equation $\dot{p}=-\partial H / \partial q$. Therefore Wu's counterexample for the inverse BGS conjecture is not acceptable, since the classical limit of a potential whose entire spectrum obeys GOE level statistics makes no physical sense. Subsequently, no meaning can be assigned to the question whether it leads to chaotic dynamics or not.

\section{MATHEMATICAL PROPERTIES OF THE $N \rightarrow \infty$ LIMIT}

In the previous section we demonstrated that a quantum potential whose energy eigenvalues follow GOE level statistics is nowhere differentiable in the $N \rightarrow \infty$ limit. Can one classify the behavior of this potential any further? Is there a characteristic width of the oscillations appearing in the potential? If 'yes', what is the asymptotic value of this characteristic width as $N$ increases?
In order to answer these questions we are going to focus on the fluctuation part of the potential and calculate for instance its numerical Fourier transform, $\mathcal{F}\left[V_{\mathrm{fl}}(x)\right]$. The Fourier components, for different values of $N$, are depicted in Fig. 8. It is apparent that the Fourier-transform differs from zero only in an interval $\left[0, f_{\max }\right]$. This upper bound can be thought of as a characteristic frequency, which translates into a characteristic width. As $N$ increases $f_{\text {max }}$ increases as well (see inset of Fig. 8) therefore waves with smaller and smaller wavelength appear in the graph of the potential. In the $N \rightarrow \infty$ limit we expect that every wavelength will appear, so the graph of the potential is going to lose its characteristic length scale. This is exactly a particular property of fractals. In Fig. 9 the fractal dimension of the potential is plotted, obtained by using the standard box-counting algorithm. Both methods employed yield similar behavior: a slowly increasing box-dimension as $N$ increases. Unfortunately the computed dimension fluctuates too much to derive a definite conclusion. However, the dimension is most likely not smaller than 1.6 in the $N \rightarrow \infty$ limit. This is comparable to the value of 1.5 obtained in an earlier work for the potential derived from the nontrivial zeros of the Riemann zeta-function [14]. This means that the potential strongly shades the complete plane for large-enough $N$, which can be seen in the inset of Fig. 9. Earlier we concluded that our potential is nowhere differentiable. Furthermore continuity would require $\overline{\Delta y}$ to decrease and approach zero as the $N \rightarrow \infty$. In Fig. 5 one can see that $\overline{\Delta y}$ does not tend to zero. If $\overline{\Delta y}$ increases indefinitely, the graph of the potential is not even bounded. In order to analyse this possibility, we also calculated the area under the graph of the potential defined as the definite integral of its absolute value $\int\left|V_{\mathrm{fl}}(x)\right| d x$. Fig. 10] shows the number of nodes, $U$, while the inset depicts the total area under the graph of the potential of the same graph. It is apparent from Fig. 10 that both the area and the number of nodes increases linearly with $N$. The area under one oscillation is the fraction of the slopes of these lines, which turns out to be a constant, irrespectively of the method we employ. Since for increasing $N$ the average width of oscillations tends to $0, \overline{\Delta x} \rightarrow 0$, but the area under one oscillation remains constant, one might conclude that the height of a wave ought to tend to infinity, i.e. $\overline{\Delta y} \rightarrow \infty$, i.e. the potential has a Dirac-delta-like singularity at every point in the $N \rightarrow \infty$ limit. In order to confirm this conclusion, we have calculated the Fouriertransform of sum of sinc-functions centered at random $x$ value. The result of this calculation showed qualitatively similar behavior as the one presented in Fig. 8 .

\section{CONCLUSIONS}

In this paper we investigated the inverse BGS conjecture and the validity of the counterexample given by $\mathrm{Wu}$ et al. The Hamiltonian of our model takes its most standard form $p^{2} / 2 m+V(x)$, and we created one-dimensional 

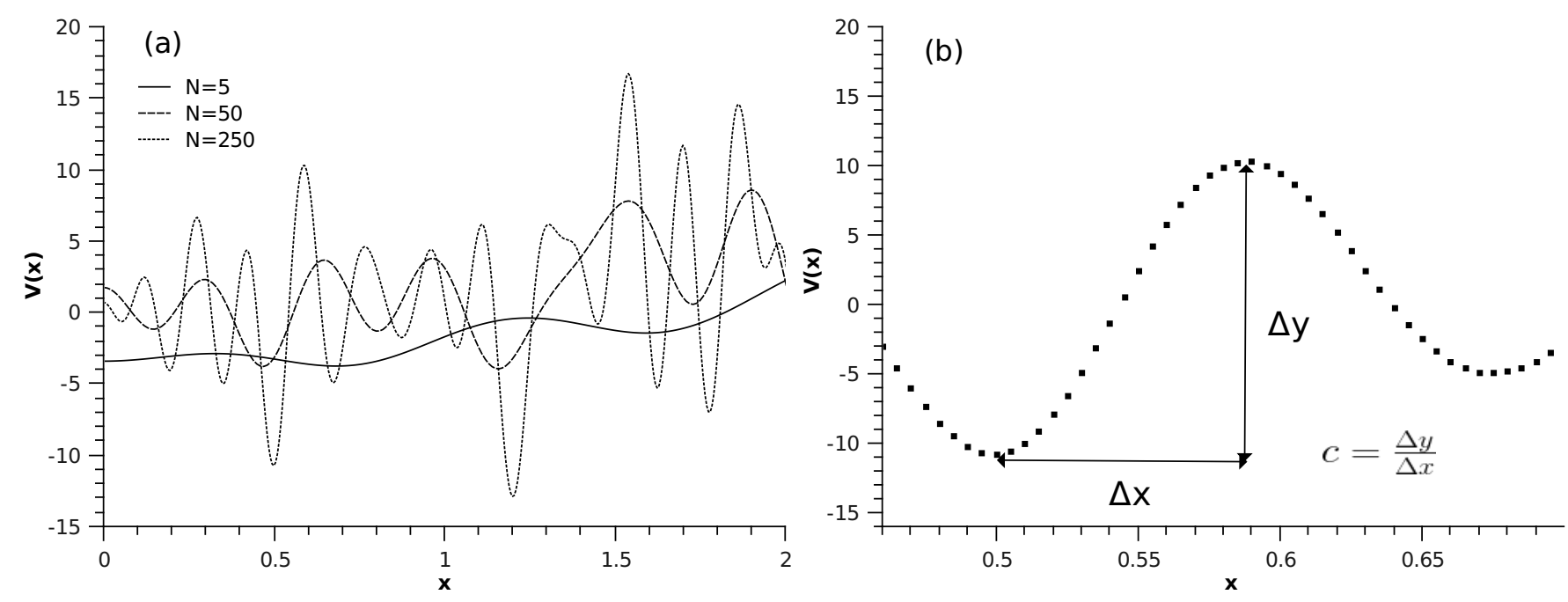

FIG. 3. (a) The potential calculated with the $\mathrm{Wu}$ method for $N=5$ (continuous line), $N=50$ (dashed line), $N=250$ (points) in the interval $[0 ; 2]$. (b) The meaning of $\Delta x$ and $\Delta y$.

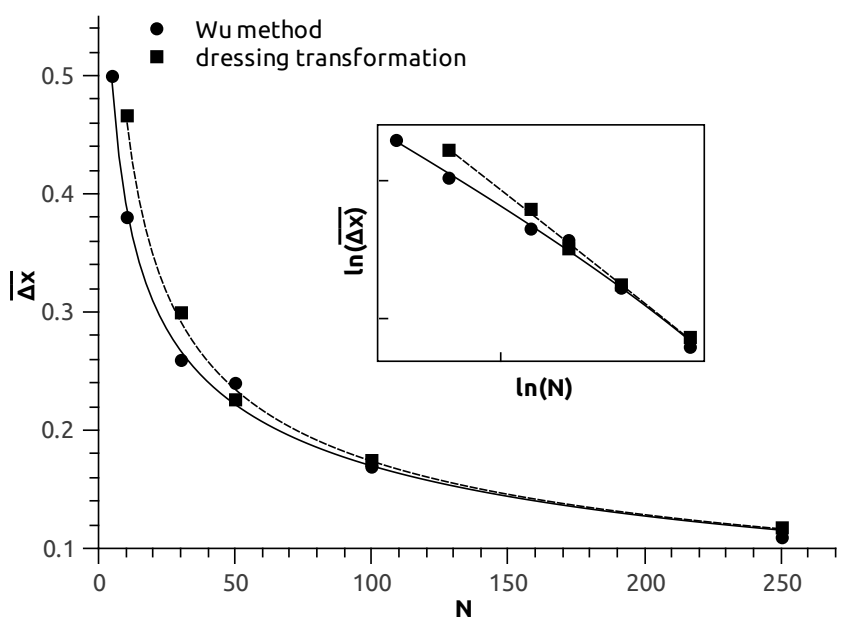

FIG. 4. The average distance of two adjacent extrema, $\overline{\Delta x}$, is plotted as a function of $N$ for both methods. The solid and dashed lines are plotted only to guide the eye and are obtained by fitting the function $A+B N^{-C}$ onto the data. The inset is the same figure on a log-log scale.

quantum potentials, $V(x)$, whose first $N$ energy levels follow GOE properties. We investigated the $N \rightarrow \infty$ limit and showed that such a potential is nowhere differentiable and nowhere continuous, moreover may it has a singularity at "every" point. No sensible physical meaning can be associated with such an ill-behaving potential, therefore, the question whether it is chaotic or not, cannot be interpreted either. We mention here, however, the logically plausible, but improbable scenario that a more complicated Hamiltonians, e.g. Harper's model $\cos (p)+\cos (x)$ [24, 25], may reproduce the GOE statistics.

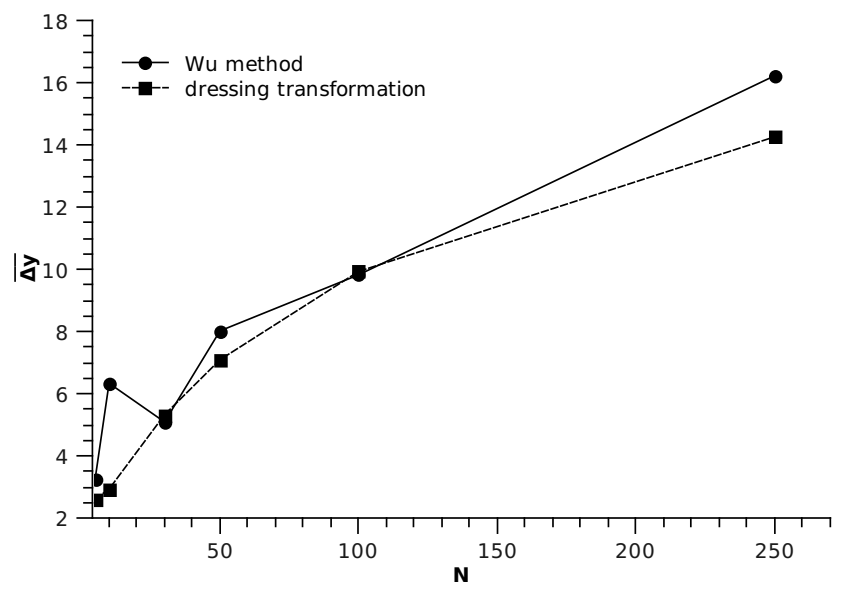

FIG. 5. The average vertical "jump" between two adjacent extrema, $\overline{\Delta y}$, is depicted as a function of $N$ obtained using both methods.

We used a method originally employed by $\mathrm{Wu}$ et al. , and the dressing transformation in order to find a potential whose entire spectrum obeys GOE statistics. These methods are based on different ideas, nevertheless they provided quantitatively the same results for the relevant measures investigated. The range of $N$ was between 5 and 250 .

We further analyzed the $N \rightarrow \infty$ limit by calculating the Fourier spectrum of the fluctuating part of the potential. We demonstrated that its Fourier components do not tend to zero at large magnitudes of $f$. Therefore the potential loses its characteristic length scale, and its structure resembles that of a fractal, the dimension is above 1.6. This finding also supports our conclusion, 


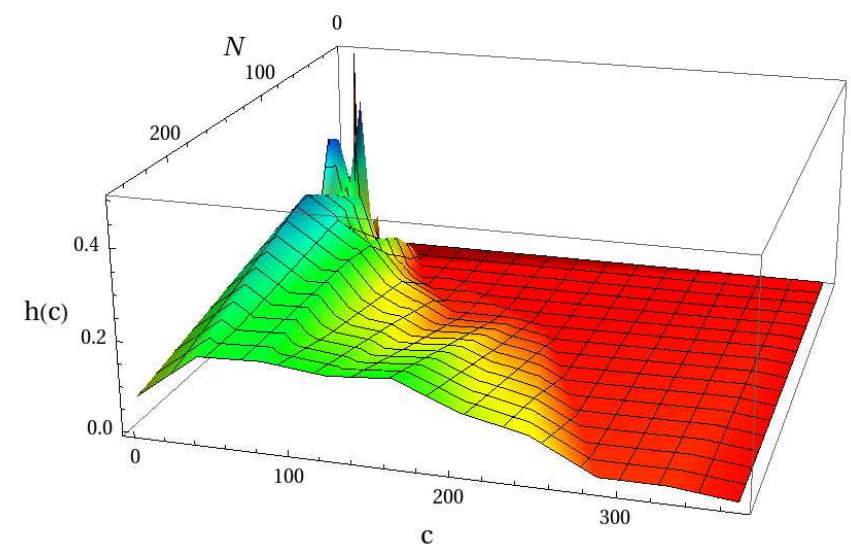

FIG. 6. (Color online) The histogram of the parameter $c$ is depicted for different values of $N$.

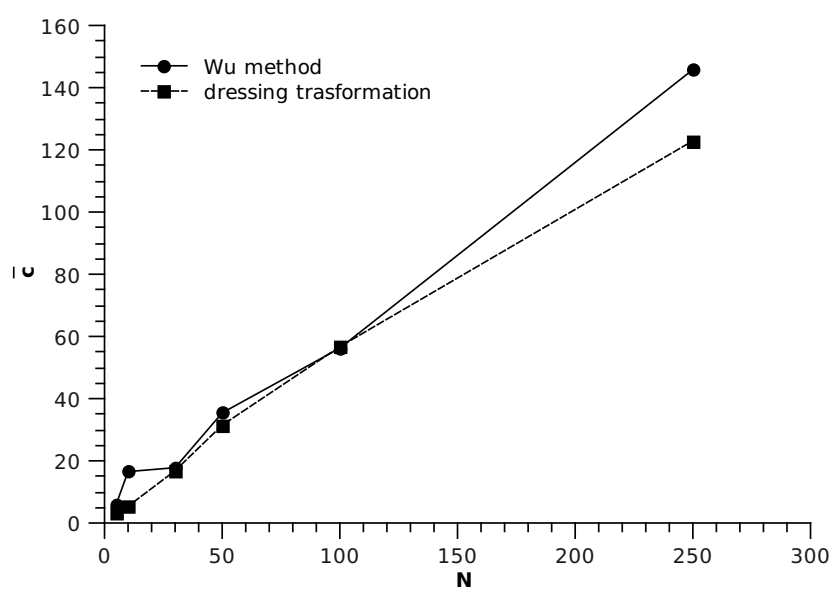

FIG. 7. The average of $c$ as a function of $N$ with the $\mathrm{Wu}$ method and the dressing transformation. namely a potential having GOE spectrum does not have a classical analogue, therefore it is not an acceptable counterexample against the inverse BGS conjecture.

\section{ACKNOWLEDGMENTS}

The authors are indebted to Dr. P. Bálint and Prof. B. Eckhardt for fruitful and enlightening discussions. The authors thank one of the referees for pointing out Refs. [4, 5, 10]. This work was partially financed by the Hungarian Research Fund (OTKA) grants K73361 and K75529, the New Hungary Development Plan (Project ID: TÁMOP-4.2.1/B-09/1/KMR2010-0002) and the Alexander von Humboldt Foundation. D. S. acknowledges the financial support by the University of Otago.
[1] O. Bohigas, M. J. Giannoni, and C. Schmit, Phys. Rev. Lett. 52, 1 (1984).

[2] The BGS conjecture contains two concepts which deserve further explanation. K-systems: In the mid-1950s Kolmogorov introduced a new measure, the metric entropy or K-entropy, to classify chaotic systems. Systems with positive K-entropy, such as Arnold's famous catmap, have finite phase space with exponentially separating orbits. These systems are called K-systems. GOE: The central objects of random matrix theory are the special ensembles of matrices. These ensembles are characterized by the size, the symmetry of the matrices and the domain of the individual random elements of these matrices, e.g. GOE is an abbreviation for the Gaussian Orthogonal Ensemble consisting real symmetric matrices for which the correlated probability of the diagonal elements is invariant under orthogonal transformation and follow a multi-dimensional normal distribution. This ensemble may, for example, correspond to spinless systems, invariant under time reversal.

[3] in Proceedings of the Les Houches Summer School of Theoretical Physics, edited by M. J. Giannoni, A. Voros, and J. Zinn-Justin ((Elsevier, New York, 1991), 1989).

[4] J. Hannay and A. M. O. de Almeida, J. Phys. A 17, 3429 (1984).

[5] M. V. Berry, Proc. Roy. Soc. Lond. A 400, 229 (1985).

[6] A. V. Andreev, O. Agam, B. D. Simons, and B. L. Altshuler, Phys. Rev. Lett. 76, 3947 (1996).

[7] A. V. Andreev, B. D. Simons, O. Agam, and B. L. Altshuler, Nuclear Physics B 482, 536 (1996).

[8] S. Müller, S. Heusler, P. Braun, F. Haake, and A. Altland, Phys. Rev. Lett. 93, 014103 (2004).

[9] S. Müller, S. Heusler, P. Braun, F. Haake, and A. Altland, Phys. Rev. E 72, 046207 (2005).

[10] J. P. Keating and S. Müller, Proc. Roy. Soc. Lond. A 463, 3241 (2007).

[11] E. B. Bogomolny, B. Georgeot, M.-J. Giannoni, and C. Schmit, Phys. Rep. 291, 219 (1997). 


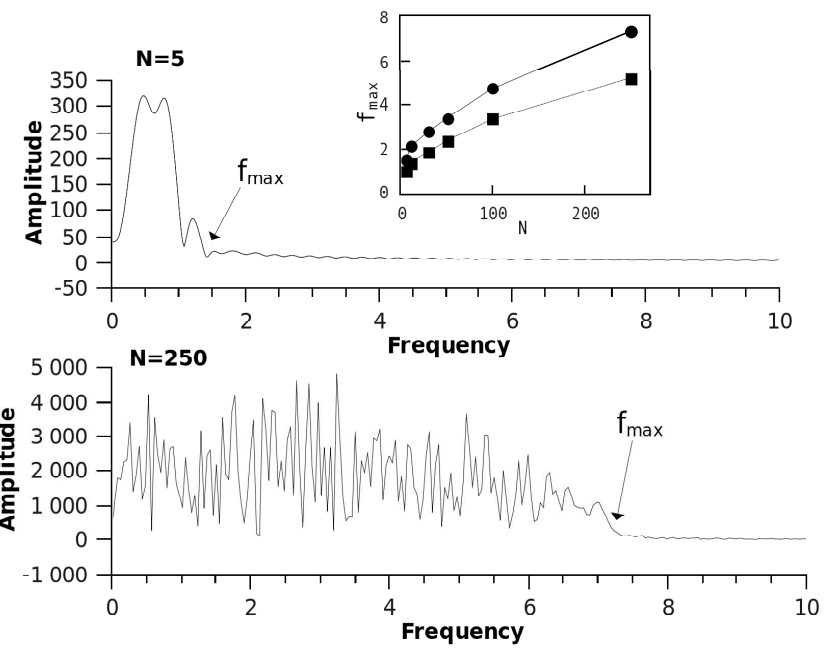

FIG. 8. The Fourier-transform of $V_{\mathrm{fl}}(x)$ at $N=5$ (upper graph) and at $N=250$ (lower graph). $\mathcal{F}\left[V_{\mathrm{f}}(x)\right]$ gets wider as $N$ increases. $\mathcal{F}\left[V_{\mathrm{fl}}(x)\right]$ vanishes beyond a characteristic wavenumber for every $N$ denoted as $f_{\max }$. Inset: $f_{\max }$ as a function of $N$ with both methods. The two curves show very similar behavior.

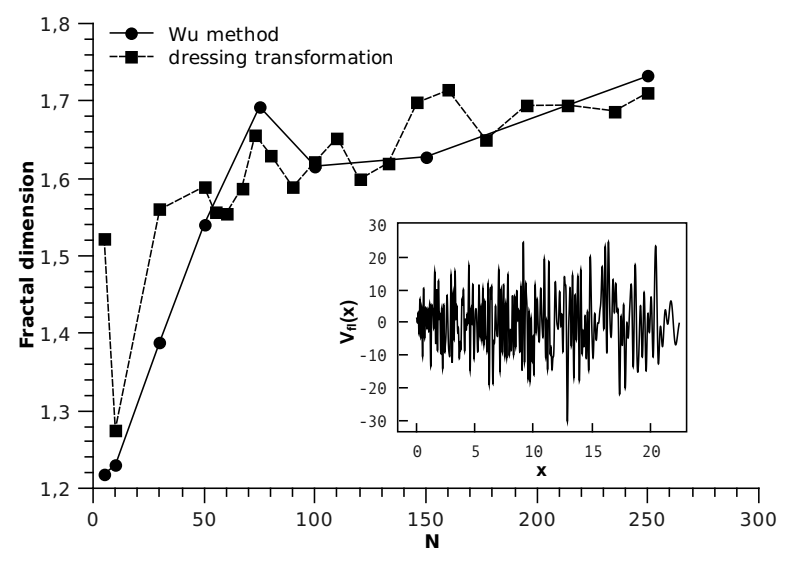

FIG. 9. The box-dimension of $V_{\mathrm{fl}}(x)$ as a function of $N$. Inset: $V_{\mathrm{fl}}(x)$ at $N=250$.

[12] H. Wu, M. Vallières, D. H. Feng, and D. W. L. Sprung,
Phys. Rev. A 42, 1027 (1990).

[13] V. I. Arnold, Mathematical methods of classical mechanics, 2nd ed., Graduate Texts in Mathematics, Vol. 60 (Springer Verlag, New York, 1989).

[14] H. Wu and D. W. Sprung, Phys. Rev. E 48, 2595 (1993).

[15] A. Ramani, B. Grammaticos, and E. Caurier, Phys. Rev. E 51, 6323 (1995).

[16] H. Wu and D. W. Sprung, Phys. Rev. E 51, 6327 (1995).

[17] B. P. van Zyl and D. A. Hutchinson, Physical Review E 67, 066211 (2003).

[18] D. Schumayer, B. P. van Zyl, and D. A. W. Hutchinson, Phys. Rev. E 78, 056215 (2008).

[19] D. Schumayer and D. A. W. Hutchinson, Rev. Mod. Phys. 83, 307 (2011).

[20] Unfolding is a standard procedure in RMT to scale the

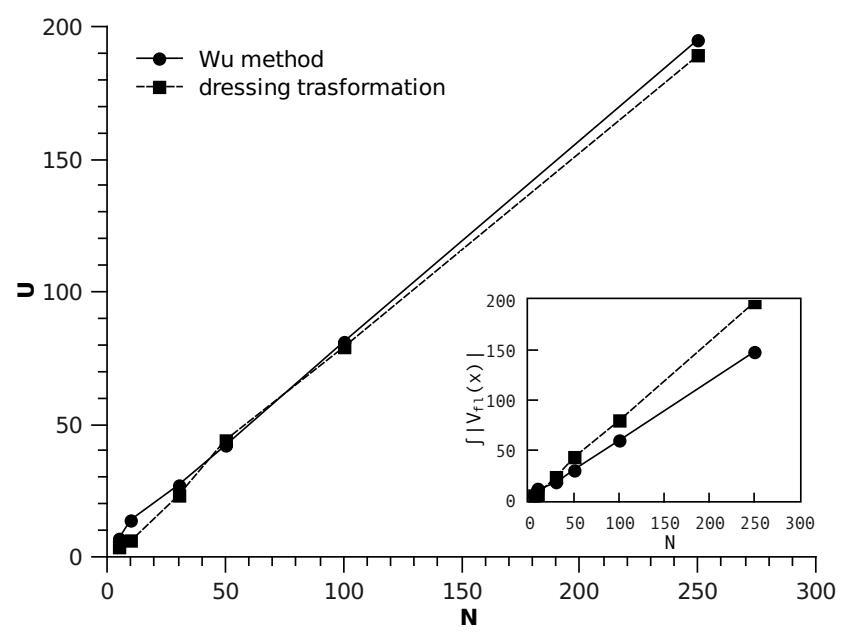

FIG. 10. The number of nodes of the potential is plotted as a function of $N$. Inset shows the absolute value of $V_{\mathrm{fl}}(x)$ integrated over the entire $x$-axis. The solid and dashed lines correspond the same method in both graphs.

mean level spacing of a given spectrum to be unity, thus different spectra become comparable.

[21] A. Shabat, Inverse Problems 8, 303 (1992).

[22] G. Borg, Acta Mathematica 78, 1 (1946).

[23] B. N. Zakhariev and A. A. Suzko, Direct and Inverse Problems: Potentials in Quantum Scattering (Springer, 1990).

[24] P. G. Harper, Proc. Phys. Soc. A 68, 874 (1955).

[25] D. R. Hofstadter, Phys. Rev. B 14, 2239 (1976). 